\title{
Let us Open Our Eyes to Swallow an Imported Lyme: A Gase of Lyme Disease
}

NV Sundarachari, U Veeramma, A Sridhar' ${ }^{1}$ KYN Varma'

From the Department of Neurology and Department of Internal Medicine';

Guntur Government Hospital, Guntur-522007, Andhra Pradesh, India.

\section{Abstract:}

Lyme disease is an arthropod borne spirocheteal disease with multisystem manifestation spread over long periods of time. Neurological features may be a clue to the diagnosis of this disease. There have been only sporadic reports of these cases in India. We present a case of lyme disease with atypical features.

Key words: Lyme Disease, Ticks, Spirochaeta, Facial Paralysis, Deglutition Disorders.

\section{Introduction}

The spectrum of neurological diseases many a times defies etiological diagnosis. In a tropical country like India, many of these vague manifestations could be due to infectious disease. Very rarely are these diseases identified or diagnosed. One such infection that can cause neurological symptoms is lyme disease caused by Borrelia burgdorferi, a spirochete transmitted by the bite of ixodes tick.

Early infection consists of localized erythema migrans (stage 1), followed within days or weeks by disseminated infection that affects the nervous system, heart, or joints in particular (stage 2) and subsequently, within weeks or months, by late or persistent infection (stage 3) [1]. We present a case of Lyme disease with atypical features and which is rarely reported in this part of the world.

\section{Case Report}

A 25 year old young woman came to us with headache since last two days. As the headache progressed she was unable to close her eyes and she couldn't smile thereafter. Mouth opening was painful to the extent she found it hard to open her mouth to quench her thirst with water or eat a morsel of food. However, there was no regurgitation or choking. Added to it was the pain over the back of the cheeks that gnawed and increased on opening her mouth. She denied any history of fever, vaccination, animal bite or a neurologic deficit in recent past. There was no history of hypertension or diabetes.

Examination was unremarkable expect for inflamed parotid glands, bilateral LMN facial palsy and blurred

\section{Corresponding Author: Dr. A. Sridhar}

Email: iamimenotu@gmail.com

Received: May 20, 2013 | Accepted: June 3, 2013 | Published Online: June 20, 2013

This is an Open Access article distributed under the terms of the Creative Commons Attribution License (creativecommons.org/licenses/by/3.0)

Conflict of interest: None declared | Source of funding: Nil | DOl: http://dx.doi.org/10.17659/01.2013.0044 
disc margins on fundoscopy. Investigations revealed normal hemogram, elevated protein levels in CSF, and positive serum IgG mumps antibodies. Nerve conduction studies were normal. The positive serum lyme lgM antibodies were consistent with acute Borrelia burgdoferi infection. She was treated with ceftriaxone $1 \mathrm{~g}$ twice daily intravenously for 2 weeks and prednisolone $40 \mathrm{mg}$ once daily (tapered over one month). Her symptoms improved with the therapy.

\section{Discussion}

Lyme disease was first reported in the United States in the town of Old Lyme, Connecticut, in 1977. The same disease occurs in many parts of Europe and Asia. There are 3 stages of Lyme disease: symptoms of early localized Lyme disease (Stage 1) are similar to the flu and there may be a flat or slightly raised red spot at the site of the tick bite with a clear area in the centre (bull's eye rash). It can be large and expanding in size. This rash is called erythema migrans. Without treatment, it can last 4 weeks or more. Symptoms of early disseminated lyme disease (Stage 2) may include, numbness or pain in the nerve area, paralysis or weakness in the muscles of the face, palpitations, chest pain, or shortness of breath. Symptoms of late disseminated lyme disease (Stage 3) include muscle and joint pain, abnormal muscle movement, joint swelling, muscle weakness, numbness and tingling, speech problems and cognitive problems [2].

We considered various differential diagnosis for this patient. Lyme disease is known to cause facial palsy and dysphagia. Parotitis in this case is an unusual manifestation. Headache and weakness are features of inflammatory reaction in the body. Positive lyme antibodies are diagnostic indicators. Meningitis with involvement of cranial nerves ought to have presented with neck stiffness, inflammatory cells in the cerebrospinal fluid and signal alterations in MR imaging. Active infection with mumps virus can present with parotitis and headache but the cranial nerve palsies are unreported. In this case, positive serum lgG antibodies for mumps virus indicate only remote infection with mumps and not active or ongoing infection. The normal nerve conduction studies ruled out the Miller Fisher variant of Guillian Barre syndrome and the normal hemogram eliminated any possibility of leukemia. There have been cases reported sporadically across various parts of India [3-5]. The combination of facial palsy and dysphagia is an atypical presentation of this disease.

\section{Conclusion}

The increase in encroachment of wild habitat may be an important factor in the increase in reports of this disease. Its early recognition and treatment is essential in view of its long term sequelae.

\section{References}

1. Steere AC. Lyme disease. N Engl J Med. $2001 ; 345: 115-125$.

2. Bethesda (MD): U.S. National Library of Medicine; c2013. Lyme disease; 2013 May 23 [cited 2012 Feb 27]; Available from: http://www.ncbi.nlm.nih.gov/pubmedhealth/PMH0002296/

3. Patial RK, Kashyap S, Bansal SK, Sood A. Lyme disease in a Shimla boy. J Assoc Physicians India. 1990;38:503-504. 
4. Handa R, Wali JP, Singh S, Aggarwal P. A prospective study of Lyme Arthritis in North India. Indian J Med Res. 1999;1 10:107-109.

5. Babu K, Murthy PR. Neuroretinitis as a manifestation of Lyme disease in South India: A case report. Ocul Immunol Inflamm. 2010;18:97-98. 